\section{Call for Papers 2017:}

\section{Können wir Sie als Autor gewinnen?}

Wirtschaftsinformatik \& Management versteht sich als hochwertige, wissenschaftlich fundierte Fachzeitschrift für den Themenbereich Wirtschaftsinformatik und relevante Aspekte der Unternehmensführung. Der Qualitätsanspruch der inhaltlichen Arbeit wird durch ein hochkarätiges Advisory Board aus Wissenschaft und Praxis gewährleistet. Top-Entscheider und renommierte Fachautoren analysieren, bewerten und schreiben über zukunftsorientierte Themen für Praktiker auf höchstem fachlichen Niveau.

\section{Können wir auch Sie zu den nachstehenden Themen} als Autor gewinnen?

\section{Fachbeiträge allgemein}

In jeder Ausgabe finden sich neben den Texten zum Heftschwerpunkt Fachbeiträge zu Themen aus dem gesamten Spektrum der Wirtschaftsinformatik. Bitte senden Sie vorab einen Abstract (maximal eine Seite) an den Chefredakteur peter.pagel@springer.com.

\section{Schwerpunktthemen}

Als Themenheft setzt Wirtschaftsinformatik \& Management in jeder Ausgabe einen inhaltlichen Schwerpunkt. Für folgende Schwerpunktthemen können Sie sich noch mit Ihrem Beitrag an die E-MailAdresse des Chefredakteurs bewerben:

\section{Heft 1 Neue Organisationsmodelle:}

Hat der CIO ausgedient?

\section{Heft 2 Komplexität}

\section{Heft 3 IT und Mobilität}

Heft 4 IT und Recht

Heft 5 Künstliche Intelligenz -

$$
\text { Deep Learning \& Co. }
$$

Heft 6 Techno-Stress - über den Umgang mit allgegenwärtiger Technologie

\section{Advisory Board}

Person $\quad$ Organisation

Dr. Udo Bub

Deutsche Telekom Laboratories

Prof. Dr. Dieter

Universität Leipzig

Ehrenberg

Prof. Dr.

Universität Siegen

Manfred

Grauer

Dr. Jürgen

Laartz

McKinsey \& Company, Inc.

Prof. Dr. Dr. h.c. Universität Erlangen-Nürnberg mult. Peter

Mertens

Prof. Dr.

Matthias

Universität Göttingen

Schumann

Prof. Dr.

Universität St. Gallen

Andrea Back

Prof. Dr. Dr. h.c. Universität Köln

Norbert

Szyperski

Prof. Dr.

Universität Kassel

Udo Winand

Dr. Peter

SAP Deutschland AG \& Co. KG

Zencke

Wirtschaftsinformatik \& Management verfolgt das Ziel, den Austausch zwischen Theorie und Praxis der Wirtschaftsinformatik zu fördern. Angestrebt ist aus diesem Grund eine ausgewogene Mischung aus forschungsnahen und Beiträgen aus der Praxis. Gemeinsam ist allen Beiträgen der Bezug zur praktischen Arbeit. 